\title{
Rationalized evaluation subgroups of mapping spaces between complex Grassmannians
}

\section{Paul Antony Otieno ${ }^{1}$ (D) Jean Baptiste Gatsinzi ${ }^{2} \cdot$ Vitalis Onyango-Otieno $^{1}$}

Received: 22 December 2018 / Accepted: 14 October 2019 / Published online: 31 October 2019

(c) The Author(s) 2019

\section{Abstract}

We determine evaluation subgroups of the inclusion $\operatorname{Gr}(2, n) \hookrightarrow \operatorname{Gr}(2, n+1)$ between complex Grassmannians.

Keywords Evaluation subgroups · Gottlieb group $\cdot G$-sequence

Mathematics Subject Classification Primary 55P62 · 54C35

\section{Introduction}

Given a pointed topological space $\left(X, x_{0}\right)$, the $n t h$ Gotttlieb group of $X$, also called the evaluation subgroup of $\pi_{n}(X)$ and denoted by $G_{n}(X)$, consists of those $\alpha \in \pi_{n}(X)$ for which there is a map $F: X \times S^{n} \longrightarrow X$ such that the following diagram commutes:

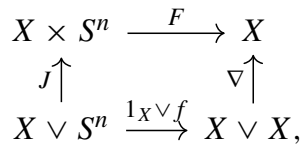

where $f: S^{n} \longrightarrow X$ is a representative of $\alpha$ and $\nabla$ is the folding map. Thus for every $\alpha \in G_{n}\left(X, x_{0}\right)$, there exists at least one map $F: X \times S^{n} \longrightarrow X$ such that $F\left(x_{0}, s\right)=f(s)$. We say that $F$ is an affiliated map to $\alpha$ [3]. If $X$ has a base point $x_{0}$ and aut $X$ denotes the monoid of self homotopy equivalences of $X$ with $e v:$ aut $X \longrightarrow X$ the evaluation map at

Paul Antony Otieno

paotieno@strathmore.edu

Jean Baptiste Gatsinzi gatsinzij@biust.ac.bw

Vitalis Onyango-Otieno

vonyango@strathmore.edu

1 Strathmore Institute of Mathematical Sciences, Strathmore University, Box 59857, Nairobi, Kenya

2 Department of Mathematics, Botswana International University of Science and Technology, Private Bag 16, Palapye, Botswana 
$x_{0}$, then it follows from the definition that

$$
G_{n}(X)=i m\left(e v_{\sharp}: \pi_{n}\left(\text { aut } X, 1_{X}\right) \longrightarrow \pi_{n}\left(X, x_{0}\right)\right) .
$$

Similarly, if $f: X \longrightarrow Y$ is a based map between simply connected $C W$ complexes and $\operatorname{map}(X, Y ; f)$, the space of maps from $X$ to $Y$ which are homotopic to $f$, then $G_{n}(Y, X ; f)=$ $\operatorname{im}\left(e v_{\sharp}: \operatorname{map}(X, Y ; f) \longrightarrow \pi_{n}(Y)\right)$ is the $n t h$ evaluation subgroup of $f$ [9]. In [10], Woo and Lee defined relative evaluation subgroups $G_{n}^{r e l}(X, Y ; f)$ and showed that they fit in a sequence

$$
\cdots \longrightarrow G_{n+1}^{r e l}(Y, X ; f) \longrightarrow G_{n}(X) \longrightarrow G_{n}(Y, X ; f) \longrightarrow \cdots
$$

called the $G$-sequence of $f$.

We use Sullivan models to compute rational relative Gottlieb groups of the inclusion $\operatorname{Gr}(2, n) \hookrightarrow \operatorname{Gr}(2, n+1)$. We refer to [4] for details and work over a field of characteristic zero in this case $\mathbb{Q}$.

Definition 1 A differential graded algebra (dga) is a graded algebra $A=\bigoplus_{n \geq 0} A^{n}$ together with a derivation $d, d=d_{n}: A^{n} \longrightarrow A^{n+1}$ such that $d \circ d=0$. Then $(A, d)$ is called a cochain algebra. A graded algebra $A$ is commutative if $a \cdot b=(-1)^{\operatorname{deg} a \cdot \operatorname{deg} b} b \cdot a$ for $a, b \in A$ [2, Chap. 3].

Definition 2 A Sullivan algebra is a commutative cochain algebra of the form $(\wedge V, d)$ where $V=\left\{V^{p}\right\}_{p \geq 2}$ and $\wedge V$ denotes the graded free commutative algebra on $V$. A Sullivan model for a commutative cochain algebra $(A, d)$ is a quasi-isomorphism $m:(\wedge V, d) \longrightarrow(A, d)$ from a Sullivan algebra $(\wedge V, d)$. A Sullivan algebra is said to be minimal if the differential is decomposable, that is, Im $d \subset \wedge^{+} V \cdot \wedge^{+} V$. Moreover, if $H^{0}(A)=\mathbb{Q}$ then $(A, d)$ has a minimal model which is unique up to isomorphism. If $X$ is a nilpotent space and $A_{P L}(X)$ the commutative differential graded algebra $(c d g a)$ of piecewise linear forms on $X$, then a Sullivan model of $X$ is a Sullivan model of $A_{P L}(X)$ [2, Chap.12].

\section{Derivation spaces and the rationalized G-sequence}

Given commutative differential graded algebras $\left(A, d_{A}\right)$ and $\left(B, d_{B}\right)$ and a map $\phi: A \longrightarrow B$, define a $\phi$-derivation of degree $n$ to be a linear map $\theta: A^{*} \longrightarrow B^{*-n}$ which satisfies $\theta(x y)=\theta(x) \phi(y)+(-1)^{n|x|} \phi(x) \theta(y)$. We only consider derivations of positive degree. Let $\operatorname{Der}_{n}(A, B ; \phi)$ denote the vector space of all $\phi$-derivations of degree $n$ for $n>0$. Define a linear map $D: \operatorname{Der}_{n}(A, B ; \phi) \longrightarrow \operatorname{Der}_{n-1}(A, B ; \phi)$ by $D(\theta)=d_{B} \circ \theta-(-1)^{|\theta|} \theta \circ d_{A}$. Then, $\left(\operatorname{Der}_{*}(A, B ; \phi), D\right)$ is a chain complex. In case $A=B$ and $\phi=1_{B}$, the chain complex of derivations $\operatorname{Der}_{*}(B, B ; 1)$ is just the usual complex of derivations on the commutative differential graded algebra $B$ [6]. If $\phi:(\wedge V, d) \longrightarrow(\wedge W, d)$ is a Sullivan minimal model of $f: X \longrightarrow Y$, then $H_{n}(\operatorname{Der}(\wedge V, \wedge W ; \phi), D) \cong \pi_{n}(\operatorname{map}(X, Y ; f)) \otimes \mathbb{Q} ; n \geq 2$ [6], [1]. We note that $\operatorname{Der}(\wedge V, B ; \phi) \cong \operatorname{Hom}(V, B)$. If $\left\{v_{i}\right\}$ is a basis of $V$, we denote by $\left(v_{i}, b\right)$, the unique $\phi$-derivation $\theta$ such that

$$
\begin{cases}\theta\left(v_{i}\right)=b_{i} & b_{i} \in B, \\ \theta\left(v_{j}\right)=0 & i \neq j .\end{cases}
$$

Pre-composition with $\phi$, respectively post-composition with the augmentation $\varepsilon: B \longrightarrow \mathbb{Q}$, gives a map of chain complexes

$$
\phi^{*}: \operatorname{Der}_{*}(B, B ; 1) \longrightarrow \operatorname{Der}_{*}(A, B ; \phi),
$$


respectively

$$
\varepsilon_{*}: \operatorname{Der}_{*}(A, B ; \phi) \longrightarrow \operatorname{Der}_{*}(A, \mathbb{Q} ; \varepsilon) .
$$

Definition 3 Let $\phi: V \longrightarrow W$ be a map of differential graded vector spaces. Define a differential graded vector space, $\operatorname{Rel}_{*}(\phi)$, called the mapping cone as follows. $\operatorname{Rel}_{n}(\phi)=$ $s V_{n-1} \oplus W_{n}$ with differential $\delta$ of degree -1 given by $\delta(s v, w)=\left(-s d_{V}(v), \phi(v)+d_{W}(w)\right)$ [7]. There are chain maps $J: W_{n} \longrightarrow \operatorname{Rel}_{n}(\phi)$ and $P: \operatorname{Rel}_{n}(\phi) \longrightarrow V_{n-1}$ defined by $J(w)=(0, w)$ and $P(s v, w)=v$. These give a short exact sequence of chain complexes

$$
0 \longrightarrow W_{*} \stackrel{J}{\longrightarrow} \operatorname{Rel}_{*}(\phi) \stackrel{P}{\longrightarrow} V_{*-1} \longrightarrow 0,
$$

which leads to a long exact sequence in homology

$$
\cdots \rightarrow H_{n+1}(\operatorname{Rel}(\phi)) \stackrel{H(P)}{\longrightarrow} H_{n}(V) \stackrel{H(\phi)}{\longrightarrow} H_{n}(W) \stackrel{H(J)}{\longrightarrow} H_{n}(\operatorname{Rel}(\phi)) \rightarrow \cdots,
$$

whose connecting homomorphism is $H(\phi)$. We refer to this sequence as the long exact homology sequence of $\phi$.

We use Theorem 3.3 of [6]

Theorem Let $f: X \longrightarrow Y$ be a map between simply connected $C W$-complexes and $\phi:(\wedge V, d) \longrightarrow(\wedge W, d)$ its Sullivan model. The long exact sequence induced by $f_{*}: \operatorname{map}(X, X ; 1) \longrightarrow \operatorname{map}(X, Y ; f)$ on rational homotopy groups is equivalent to the long exact homology sequence of the map

$$
\phi^{*}: \operatorname{Der}_{*}(\wedge W, \wedge W ; 1) \longrightarrow \operatorname{Der}_{*}(\wedge V, \wedge W ; \phi)
$$

induced by the minimal model $\phi:(\wedge V, d) \longrightarrow(\wedge W, d)$ of the map $f: X \longrightarrow Y$.

Definition 4 Given a commutative differential graded algebra map $\phi: A \longrightarrow B$, we have the following commutative diagram of differential graded vector spaces;

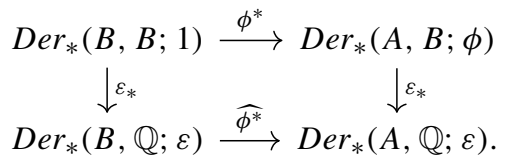

Here, $\varepsilon$ denotes the augmentation of either $A$ or $B$. On passing to homology and using the naturality of the mapping cone construction, we obtain the following homology ladder $(n \geq 2)$,

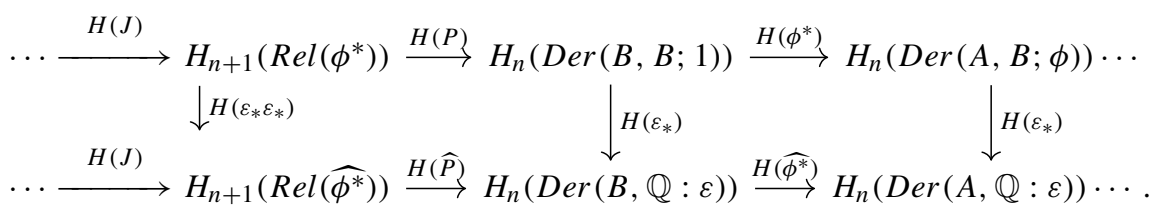

Definition 5 Suppose $\phi: A \longrightarrow B$ is a map of commutative differential graded algebras, we define the evaluation subgroups of $\phi$ by

$$
G_{n}(A, B ; \phi)=\operatorname{im}\left\{H\left(\varepsilon_{*}\right): H_{n}(\operatorname{Der}(A, B ; \phi)) \longrightarrow H_{n}(\operatorname{Der}(A, \mathbb{Q} ; \varepsilon))\right\} .
$$

In the special case $A=B$ and $\phi=1_{B}$, we refer to the Gottlieb group of $B$, and use the notation $G_{n}(B)$. If $B=(\wedge V, d)$ is a model of a simply connected space $X$, then an element 
$\alpha \in G_{n}(B)$ is represented by a linear mapping $f: V^{n} \longrightarrow \mathbb{Q}$ that extends into a derivation $\theta$ of $\wedge V$ such that $\delta \theta=0$. Moreover, $G_{n}(B) \cong G_{n}\left(X_{\mathbb{Q}}\right)$ [2, Proposition 29.8].

The $n t h$ relative evaluation subgroup of $\phi$ is defined by;

$$
G_{n}^{r e l}(A, B ; \phi)=\operatorname{im}\left\{H\left(\varepsilon_{*}, \varepsilon_{*}\right): H_{n}\left(\operatorname{Rel}\left(\phi^{*}\right)\right) \longrightarrow H_{n}\left(\operatorname{Rel}\left(\widehat{\phi^{*}}\right)\right)\right\} .
$$

Then the image of the upper long sequence in the lower, of the ladder above, gives a sequence

$$
\cdots \stackrel{H(\widehat{J})}{\longrightarrow} G_{n+1}^{r e l}(A, B ; \phi) \stackrel{H(\widehat{P})}{\longrightarrow} G_{n}(B) \stackrel{H\left(\widehat{\phi}^{*}\right)}{\longrightarrow} G_{n}(A, B ; \phi) \stackrel{H(\widehat{J})}{\longrightarrow} \cdots
$$

that terminates in $G_{2}(A, B ; \phi)$.

We refer to this sequence as the $G$-sequence of the map $\phi: A \longrightarrow B$. This can be applied to the minimal model $\phi:(\wedge V, d) \longrightarrow(\wedge W, d)$ of the map $f: X \longrightarrow Y$ as stated and proved in [6, Theorem 3.5].

\section{The inclusion $\operatorname{Gr}(2, n) \hookrightarrow \operatorname{Gr}(2, n+1)$}

Let $\operatorname{Gr}(k, n)$ be the Grassmann manifold of $k$-dimensional subspaces of $\mathbb{C}^{n}$. The cohomology ring $H^{*}(G r(k, n), \mathbb{Q})$ is generated by the Chern classes $c_{i} \in H^{2 i}(G r(k, n), \mathbb{Q})$, for $1 \leq i \leq$ $k$. Further, the cohomology ring has a presentation

$$
H^{*}(G r(k, n), \mathbb{Q})=\wedge\left(c_{1}, c_{2}, \ldots, c_{k}\right) /\left(h_{n-k+1}, \ldots, h_{n}\right),
$$

as the quotient of the polynomial ring generated by $c_{1}, c_{2}, \ldots, c_{k},\left|c_{i}\right|=2 i$, modulo the ideal generated by the elements $h_{j}, n-k+1 \leq j \leq n$. Here, $h_{j}$ is defined as the $2 j t h$ degree term in the Taylor's expansion of $\left(1+c_{1}+c_{2}+c_{3}+\cdots+c_{k}\right)^{-1}$ where $\left(1+c_{1}+c_{2}+c_{3}+\cdots+c_{k}\right)$ is the total Chern class [4].

In particular, the cohomology rings of $\operatorname{Gr}(2, n)$ and $\operatorname{Gr}(2, n+1)$ are:

$$
\begin{aligned}
& H^{*}(G r(2, n), \mathbb{Q})=\wedge\left(y_{2}, y_{4}\right) /\left(h_{n-1}, h_{n}\right) \text { and } \\
& H^{*}(\operatorname{Gr}(2, n+1), \mathbb{Q})=\wedge\left(x_{2}, x_{4}\right) /\left(h_{n}, h_{n+1}\right) \text { respectively. }
\end{aligned}
$$

The minimal model of $\operatorname{Gr}(2, n)$ is $\left(\wedge\left(y_{2}, y_{4}, y_{2 n-3}, y_{2 n-1}\right), d\right)$ with $d\left(y_{2}\right)=d\left(y_{4}\right)=0$, $d\left(y_{2 n-3}\right)=h_{n-1}, d\left(y_{2 n-1}\right)=h_{n}$. In the same way, a model of $\operatorname{Gr}(2, n+1)$ is given by $\left(\wedge\left(x_{2}, x_{4}, x_{2 n-1}, x_{2 n+1}\right), d\right)$ with $d x_{2}=d x_{4}=0, d x_{2 n-1}=h_{n}$ and $d x_{2 n+1}=h_{n+1}$.

Lemma $1 h_{n+1}=-x_{2} h_{n}-x_{4} h_{n-1}$.

Proof Write the Taylor series $\left(1+x_{2}+x_{4}\right)^{-1}=1+h_{1}+h_{2}+\cdots$ where, $\left|h_{i}\right|=2 i$. From $\left(1+x_{2}+x_{4}\right)\left(1+x_{2}+x_{4}\right)^{-1}=1$, one gets the relation $h_{n+1}=-x_{2} h_{n}-x_{4} h_{n-1}$

In particular, $h_{n+1}$ is co-boundary in $\left(\wedge\left(x_{2}, x_{4}, x_{2 n-3}, x_{2 n-1}\right), d\right)$, that is, there exists $\alpha$ of degree $2 n+1$ such that $d \alpha=h_{n+1}$.

Theorem 1 Let $B=\left(\wedge\left(y_{2}, y_{4}, y_{2 n-3}, y_{2 n-1}\right), d\right)$. Then $G_{n}(B)=\left\langle\left[y_{2 n-3}^{*}\right],\left[y_{2 n-1}^{*}\right]\right\rangle$.

Proof Let $\alpha_{2 n-1}=\left(y_{2 n-1}, 1\right)$ and $\alpha_{2 n-3}=\left(y_{2 n-3}, 1\right)$. Then $\delta \alpha_{n-1}=\delta \alpha_{2 n-3}=0$. Moreover, $\alpha_{2 n-3}$ and $\alpha_{2 n-1}$ can not be boundaries for degree reason. Therefore, $\left[\alpha_{2 n-3}\right]$ and $\left[\alpha_{2 n-1}\right]$ are non zero homology classes in $H_{*}(\operatorname{Der}(B, B ; 1))$. Further, $\varepsilon_{*}\left(\alpha_{2 n-3}\right)=y_{2 n-3}^{*}$ and $\varepsilon_{*}\left(\alpha_{2 n-1}\right)=y_{2 n-1}^{*}$.

As $\operatorname{Gr}(2, n)$ is a finite $C W$-complex then $G_{\text {even }}(B)=0$ [2, Pg.379] . Hence, $G_{n}(B)=$ $\left\langle\left[y_{2 n-3}^{*}\right],\left[y_{2 n-1}^{*}\right]\right\rangle$. 
The inclusion $\operatorname{Gr}(2, n) \hookrightarrow G r(2, n+1)$ has a model of the form

$$
\phi: \wedge V=\left(\wedge\left(x_{2}, x_{4}, x_{2 n-1}, x_{2 n+1}\right), d\right) \longrightarrow\left(\wedge\left(y_{2}, y_{4}, y_{2 n-3}, y_{2 n-1}\right), d\right)=B
$$

where $\phi\left(x_{2}\right)=y_{2}, \phi\left(x_{4}\right)=y_{4}, \phi\left(x_{2 n-1}\right)=y_{2 n-1}$ and $\phi\left(x_{2 n+1}\right)=\alpha$ where $d \alpha=h_{n+1}$ by Lemma 1.

Theorem 2 Consider the inclusion $\operatorname{Gr}(2, n) \hookrightarrow G r(2, n+1)$ and $\phi:(\wedge V, d) \longrightarrow(B, d)$ its Sullivan model, then $G_{*}(\wedge V, B ; \phi) \cong\left\langle\left[x_{2 n-1}^{*}\right],\left[x_{2 n+1}^{*}\right]\right\rangle$.

Proof As $\operatorname{Gr}(2, n)$ is formal, $\operatorname{Der}(\wedge V, B ; \phi) \stackrel{\simeq}{\longrightarrow} \operatorname{Der}(\wedge V, H(B) ; f \circ \phi)$ where $f$ : $B \stackrel{\simeq}{\rightarrow} H(B)$ is a quasi isomorphism. Similarly, since $B$ is formal $\operatorname{Der}(B, B ; 1) \simeq$ $\operatorname{Der}(B, H(B) ; f)$. Define $\theta_{2 n-1}=\left(x_{2 n-1}, 1\right), \theta_{2 n+1}=\left(x_{2 n+1}, 1\right)$ in $\operatorname{Der}(\wedge V, H(B) ; f \circ$ $\phi)$. Then $\delta \theta_{2 n-1}=\delta \theta_{2 n+1}=0$. Moreover, $\left[\theta_{2 n-1}\right]$ and $\left[\theta_{2 n+1}\right]$ are nonzero cohomology classes in $H_{*}\left(\operatorname{Der}\left(\wedge V, H^{*}(B) ; f \circ \phi\right)\right)$.

We note that, $\theta_{2}=\left(x_{2}, 1\right)$ and $\theta_{4}=\left(x_{4}, 1\right)$ are not cycles in $\operatorname{Der}(\wedge V, H(B) ; f \circ \phi)$ [8].

Further, $H\left(\varepsilon_{*}\right)\left(\left[\theta_{2 n-1}\right]\right)=\left[x_{2 n-1}^{*}\right] \in G_{2 n-1}(\wedge V, B ; f \circ \phi)$. In a similar way, $H\left(\varepsilon_{*}\right)\left(\left[\theta_{2 n+1}\right]\right)=$ $\left[x_{2 n+1}^{*}\right] \in G_{2 n+1}(\wedge V, B ; f \circ \phi)$. It then follows that $G_{*}(\wedge V, B ; f \circ \phi)=\left\langle\left[x_{2 n-1}^{*}\right],\left[x_{2 n+1}^{*}\right]\right\rangle$.

Theorem 3 Consider the inclusion $\operatorname{Gr}(2, n) \hookrightarrow G r(2, n+1)$ and

$$
\begin{aligned}
\phi:(\wedge V, d) & =\left(\wedge\left(x_{2}, x_{4}, x_{2 n-1}, x_{2 n+1}\right), d\right) \\
& \longrightarrow\left(\wedge V\left(y_{2}, y_{4}, y_{2 n-3}, y_{2 n-1}\right), d\right)=B
\end{aligned}
$$

its Sullivan model, then $G_{*}^{r e l}(\wedge V, B ; \phi)=\left\langle\left[\left(x_{2 n-3}^{*}, 0\right)\right],\left[\left(0, y_{2 n+1}^{*}\right)\right]\right\rangle$.

Proof Consider the diagram below [6].

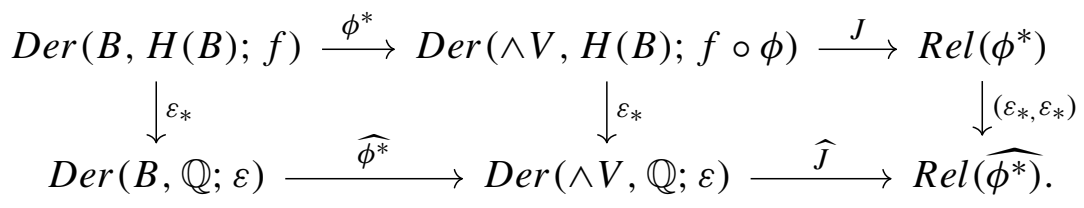

Let $\alpha_{2 n-1}=\left(y_{2 n-1}, 1\right), \alpha_{2 n-3}=\left(y_{2 n-3}, 1\right) \in \operatorname{Der}(B, H(B) ; f)$ and $\theta_{2 n-1}, \theta_{2 n+1} \in$ $\operatorname{Der}\left(\wedge V, H^{*}(B) ; \phi\right)$ as defined above. Then $\phi^{*}\left(\alpha_{2 n-1}\right)=\theta_{2 n-1}$ and $\phi^{*}\left(\alpha_{2 n-3}\right)=0$.

Further, $D\left(\alpha_{2 n-1}, 0\right)=\left(0, \theta_{2 n-1}\right), D\left(\alpha_{2 n-3}, 0\right)=(0,0)$ and $D\left(0, \theta_{2 n-1}, 0\right)=0=$ $D\left(0, \theta_{2 n+1}\right)$. Therefore, $\left[\left(\alpha_{2 n-3}, 0\right)\right]$ and $\left[\left(0, \theta_{2 n+1}\right)\right]$ are non zero homology classes in $H_{*}\left(\operatorname{Rel}\left(\phi^{*}\right)\right)$. Moreover, $H\left(\varepsilon_{*}, \varepsilon_{*}\right)\left(\left[\alpha_{2 n-3}, 0\right]\right)=\left[\left(x_{2 n-3}^{*}, 0\right)\right]$ and $H\left(\varepsilon_{*}, \varepsilon_{*}\right)\left(\left[\left(0, \theta_{2 n+1}\right)\right]\right)=$ $\left[\left(0, y_{2 n+1}^{*}\right)\right]$. A straightforward computation shows that $\left[\left(x_{2 n-3}^{*}, 0\right)\right]$ and $\left[\left(0, y_{2 n+1}^{*}\right)\right]$ span $H\left(\varepsilon_{*}, \varepsilon_{*}\right)$. 
The $G$-sequence reduces to

$$
\begin{gathered}
0 \longrightarrow G_{2 n+1}(\wedge V, B ; \phi) \stackrel{H(J)}{\simeq} G_{2 n+1}^{r e l}(\wedge V, B ; \phi) \longrightarrow 0 \cdots \\
\cdots 0 \longrightarrow G_{2 n-1}(B) \frac{H\left(\phi^{*}\right)}{\simeq} G_{2 n-1}(\wedge V, B ; \phi) \longrightarrow 0 \cdots \\
\cdots 0 \longrightarrow G_{2 n-2}^{r e l}(\wedge V, B ; \phi) \stackrel{H(P)}{\simeq} G_{2 n-3}(B) \longrightarrow 0 .
\end{gathered}
$$

and is exact.

Example 1 Consider $\operatorname{Gr}(2,4) \hookrightarrow \operatorname{Gr}(2,5)$. A model of the inclusion is given by

$$
\phi: \wedge V=\left(\wedge\left(x_{2}, x_{4}, x_{7}, x_{9}\right), d\right) \longrightarrow\left(\wedge\left(y_{2}, y_{4}, y_{5}, y_{7}\right), d\right)=B,
$$

where $d x_{2}=d x_{4}=0, d x_{7}=x_{4}^{2}-3 x^{2} x_{4}+x_{2}^{4}, d x_{9}=4 x_{2}^{3} x_{4}-3 x_{2} x_{4}^{2}-x_{2}^{5} d y_{2}=d y_{4}=0$, $d y_{5}=2 y_{2} y_{4}-y_{2}^{3}$ and $d y_{7}=y_{4}^{2}-3 y_{2}^{2} y_{4}+y_{2}^{4}$.

Moreover, $\phi\left(x_{2}\right)=y_{2}, \phi\left(x_{4}\right)=y_{4}, \phi\left(x_{7}\right)=y_{7}$ and $\phi\left(x_{9}\right)=-y_{2} y_{7}-y_{4} y_{4}$.

We compute $G_{*}^{r e l}(\wedge V, B ; \phi)$. Let $\alpha_{7}=\left(y_{7}, 1\right), \alpha_{5}=\left(y_{5}, 1\right) \in \operatorname{Der}_{7}(B, H(B) ; f)$ where $f: B \stackrel{\cong}{\rightarrow} H(B)$ and $\theta_{7}=\left(x_{7}, 1\right), \theta_{9}=\left(x_{9}, 1\right) \in \operatorname{Der}\left(\wedge V, H^{*}(B) ; f \circ \phi\right)$ then $\phi^{*}\left(\alpha_{7}\right)=\theta_{7}$ and $\phi^{*}\left(\alpha_{5}\right)=0$. Moreover, $D\left(s \alpha_{7}, 0\right)=\left(0, \theta_{7}\right), D\left(s \alpha_{5}, 0\right)=(0,0)$ and $D\left(0, \theta_{7}\right)=$ $D\left(0, \theta_{9}\right)=(0,0)$. Hence $\left[\left(s \alpha_{5}, 0\right)\right]$ and $\left[\left(0, \theta_{9}\right)\right]$ are non zero homology classes. Moreover, $\left(\epsilon_{*}, \epsilon_{*}\right)\left(s \alpha_{5}, 0\right)=\left(s y_{5}^{*}, 0\right),\left(\epsilon_{*}, \epsilon_{*}\right)\left(0, \theta_{9}\right)=\left(0, y_{9}^{*}\right)$. Therefore

$$
G_{*}^{r e l}(\wedge V, B ; \phi)=\left\langle\left[\left(0, x_{9}^{*}\right)\right],\left[\left(s y_{5}^{*}, 0\right)\right]\right\rangle .
$$

Open Access This article is distributed under the terms of the Creative Commons Attribution 4.0 International License (http://creativecommons.org/licenses/by/4.0/), which permits unrestricted use, distribution, and reproduction in any medium, provided you give appropriate credit to the original author(s) and the source, provide a link to the Creative Commons license, and indicate if changes were made.

\section{References}

1. Buijs, U., Murillo, A.: The rational homotopy Lie algebra of function spaces. Comment. Math. Helv. 83(4), 723-739 (2008)

2. Félix, Y., Halperin, S., Thomas, J.-C.: Rational Homotopy theory, Graduate Texts in Mathematics, vol. 205. Springer, New York (2001)

3. Gottlieb, D.H.: Evaluation subgroups of homotopy groups. Am. J. Math. 91, 729-756 (1969)

4. Hoffman, M.: Endomorphisms of the cohomology of complex Grassmannians. Trans. Am. Math. Soc. 281(2), 745-760 (1984)

5. Lee, K.-Y., Mimura, M., Woo, M.H.: Gottlieb groups of homogeneous spaces. Topol. Appl. 145(1-3), 147-155 (2004)

6. Lupton, G., Smith, S.B.: Rationalized evaluation subgroups of a map I: Sullivan models, derivations and $G$-sequences. J. Pure Appl. Algebra 209(1), 159-171 (2007)

7. MacLane, S.: Homology. Springer, New York (2012)

8. Otieno, P.A., Gatsinzi, J.-B., Otieno, V.O.: Rational homotopy type of mapping spaces between complex Grassmannian. Quaest. Math

9. Woo, M.H., Kim, J.-R.: Certain subgroups of homotopy groups. J. Korean Math. Soc. 21(2), 109-120 (1984) 
10. Woo, H.H., Lee, K.Y.: On the relative evaluation subgroups of a CW-pair. J. Korean Math. Soc. 25, 149-160 (1988)

Publisher's Note Springer Nature remains neutral with regard to jurisdictional claims in published maps and institutional affiliations. 